\title{
Intra-Operative Rupture of Remote Aneurysm during Transphenoidal Resection of Pituitary Macroadenoma
}

\author{
Gary Rajah, Mark Hornyak* \\ Department of Neurosurgery, Wayne State University, Detroit, USA \\ Email: grajah@med.wayene.du, ${ }^{*}$ mhornyak@med.wayne.edu
}

Received 23 January 2014; revised 23 February 2014; accepted 3 March 2014

Copyright (C) 2014 by authors and Scientific Research Publishing Inc.

This work is licensed under the Creative Commons Attribution International License (CC BY). http://creativecommons.org/licenses/by/4.0/

(c) (7) Open Access

\begin{abstract}
Rupture of intracranial aneurysm resulting in subarachnoid hemorrhage is well known to carry significant risk of poor outcome. Intra-operative rupture of an unknown intracranial aneurysm during a neurosurgical procedure is a rare occurrence that can lead to devastating consequences if not recognized and managed appropriately. Here we describe to our knowledge, the first reported case of previously unknown, remote from the surgical site, intra-operative posterior cerebral artery aneurysm rupture during transnasal endoscopic transphenoidal resection of a pituitary macroadenoma, review relevant literature, and discuss strategies to reduce surgical risk and improve patient outcomes.
\end{abstract}

\section{Keywords}

Remote Aneurysm, Rupture, Transphenoidal, Endoscope, Adenoma

\section{Introduction}

The relationship between intracranial aneurysms and pituitary adenomas has been well described. Many studies have reported a relationship between these distinct pathologies exists [1] [2], while a more recent study described their coexistence as due to chance [3]. It is generally accepted that intracranial aneurysms occur in up to $5 \%$ of the general population [3] [4], while pituitary adenomas may be found in up to $16 \%$ of the population [5]. With a significant number of patients harboring both entities, it is important to quickly recognize intra-operative rupture of a remote aneurysm during transphenoidal surgery for pituitary adenoma.

\footnotetext{
*Corresponding author.
}

How to cite this paper: Rajah, G. and Hornyak, M. (2014) Intra-Operative Rupture of Remote Aneurysm during Transphenoidal Resection of Pituitary Macroadenoma. Open Journal of Modern Neurosurgery, 4, 64-68. 


\section{Case}

A 68-year old woman presented for outpatient neurosurgical consultation with chief complaint of visual loss. She reported several months of declining vision. She was referred for MR imaging which revealed a pituitary macroadenoma with suprasellar extension causing compression of the optic chiasm (Figure 1). She had no other symptoms. She had a history of hypertension and hypothyroidism for which she was taking lisinopril and thyroid hormone. Physical examination was significant for right temporal field visual cut. Laboratory investigations revealed low TSH levels. She underwent elective endoscopic transphenoidal resection of the pituitary tumor. The case was initially unremarkable; the tumor was soft and easily removed with suction and curettage. When the tumor was nearly completely removed, the suprasellar arachnoid was draped down into the sella turcica. To better view the interior of the sella and confirm complete resection of the tumor, all instruments were removed from the field to change the endoscopic lens. When the endoscope was reintroduced, the arachnoid was herniating into the sphenoid sinus; it was tense and had a bright red color. The subarachnoid hemorrhage was immediately recognized and the arachnoid was opened. Initially bloody fluid was encountered under high pressure, but with evacuation, the pressure was relieved and the blood could be seen arising from a suprasellar source. The bleeding was controlled with irrigation and minimal packing of the sella; blood loss was estimated at $300 \mathrm{~mL}$. At the completion of the procedure she was noted to have a dilated left pupil. She was taken for emergent CT which showed diffuse SAH, then cerebral angiogram which revealed a saccular $2 \mathrm{~mm}$ left posterior cerebral artery aneurysm at the P1-P2 junction (Figure 2). The aneurysm was treated with coil embolization. The patient recovered to her baseline mental status in the intensive care unit, but suffered from a left oculomotor nerve palsy.

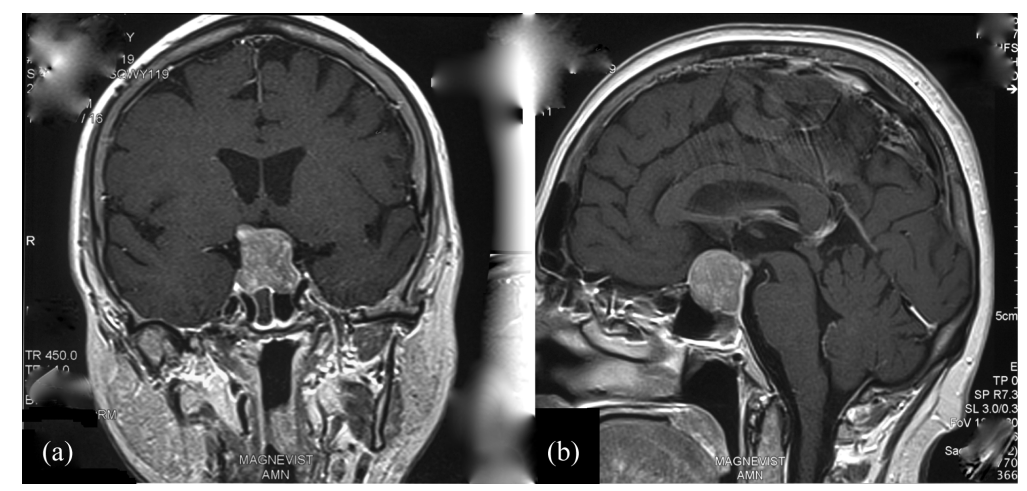

Figure 1. Coronal (a) and sagittal (b) T1-weighted, postcontrast MR images through the sella turcica demonstrating heterogeneous tumor filling sella turcica with suprasellar extension causing displacement of the optic chiasm.

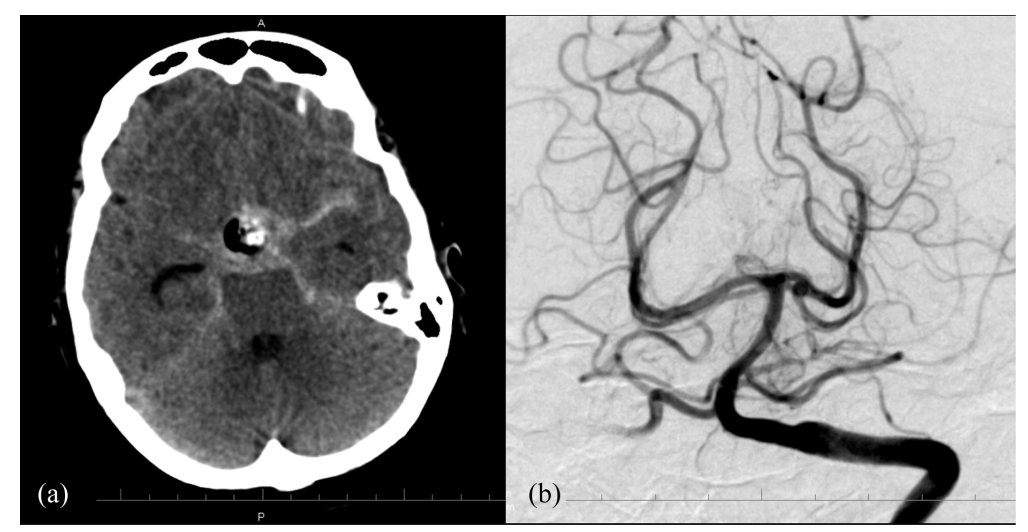

Figure 2. CT of the head (a) performed after transphenoidal resection of pituitary tumor demonstrating postoperative changes in the suprasellar region and diffuse, basilar subarachnoid hemorrhage. Left vertebral artery injection of cerebral angiogram (b) demonstrating a $2 \mathrm{~mm}$ saccular aneurysm arising from the P1-P2 segment of the left posterior cerebral artery. 
She underwent endoscopic removal of nasal packing on postoperative day 6, and she was discharged to inpatient rehabilitation without further complication on post operative day 12 with persistent third cranial nerve palsy. In followup, the patient's diplopia resolved within 3 months. At most recent followup, more than 3 years after treatment, she remains without neurological deficit, repeat angiogram is without recurrence of the aneurysm and MRI shows no tumor recurrence (Figure 3); she remains on hydrocortisone and thyroid hormone.

\section{Discussion}

Many reports have hypothesized a relationship between pituitary adenomas and aneurysms. One study found a $13.8 \%$ incidence of IA with growth hormone producing adenomas [2]. A later report suggests the incidence of IA associated with pituitary adenoma is somewhere between $3.7 \%-7.4 \%$ and $97 \%$ of these were in anterior circulation [3]. Aneurysms associated with pituitary tumors seem to be located more frequently in the anterior circulation e.g. ICA and AcoA, potentially because these vessels supply the adenoma [6]. A recent study found increasing age of the patient and cavernous sinus invasion by the tumor to be associated with increased risk of IA [7]. Proposed mechanisms for the coexsistence of these two entities include direct mechanical effects [2], circulatory changes [8], endocrine mediated processes such as diabetes, hypertension, and direct hormonal interactions with vasculature [2].

Intra-operative rupture of aneurysms during open aneurismal surgical repair is a well reported complication affecting $6.7 \%$ of aneurysms in one retrospective review [9]. Spontaneous aneurysm rupture has been reported occurring during labor [10], cervical spine surgery [11], and after blunt head trauma [12]. We have only been able to identify one other report of intra-operative hemorrhage during transphenoidal surgery due to rupture of an unknown ACOM aneurysm [13]. Other reports of intra-op aneurysm rupture during macroadenoma removal have been described, however the bulk of these are ICA aneurysms directly manipulated during hypophesectomy [14] [15]. In the three previous cases the aneurysms were found in the anterior circulation.

Etiologies of intra-operative ruptures during transphenoidal surgery vary. Direct trauma to an unidentified or hidden aneurysm by surgical instruments can damage aneurysms in the vicinity of the sella. Indirect mechanisms of rupture are largely responsible for remote ruptures such as described in this case report. Etiologies of indirect ruptures include csf egress and changes in trans mural pressure. This is supported by a link between ventricular drainage and aneurysm rupture [16]. Other etiologies include intra-operative hypertension, changes in flow dynamics, traction on arachnoid bands, shift of intracranial contents during bone or dural opening as well as removal of a space occupying lesion. One study of open craniotomies for aneurysms reported $8.3 \%$ of ruptures occurred during dural and arachnoid opening, $12.5 \%$ during hematoma evacuation, $16.7 \%$ during brain retraction and $62 \%$ during aneurysm dissection [17]. The rupture in our case likely occurred due to brain shift from the resulting decompression of the sella.

Intra-operative identification of the hemorrhage in our report was possible by viewing a tense, red diaphragm sella with the endoscope. The only other similar case report we could identify was an ACOM aneurysm rupture during macroadenoma resection [13]. However in this report the rupture was not found until after the patient failed to recover from anesthesia. Tsuchida hypothesized that the tumor's suprasellar extension abutted the ACOM and its abrupt collapse during debulking were at fault [13].

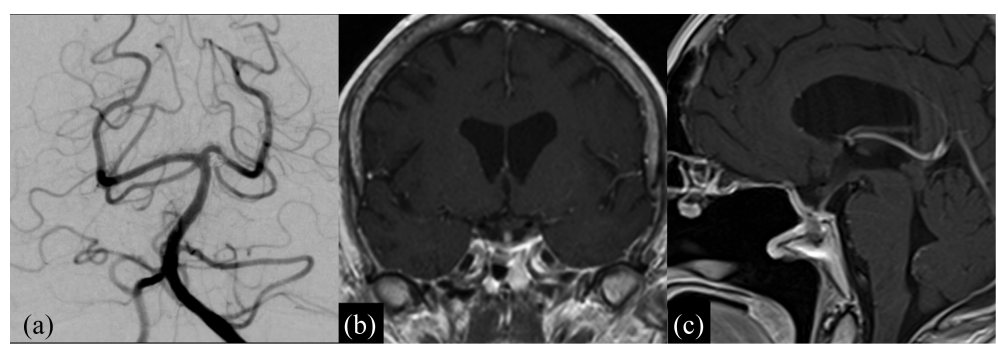

Figure 3. Left vertebral artery injection of cerebral angiogram (a) performed 2 years after coil embolization of posterior cerebral artery aneurysm demonstrating coil mass in aneurysm and stable appearance without sign of recurrence. Postcontrast T1-weighted MR images in coronal (a) and sagittal (b) planes demonstrating postoperative changes without evidence of recurrence of pituitary adenoma three years after resection. 
Management of aneurismal rupture during transphenoidal surgery requires identification, hemostasis, and angiography. The minimally invasive opening created during endoscopic surgery becomes an immediate obstacle when faced with vascular complications. All intra-operative ruptures discussed in the literature review and our report were immediately tamponaded by packing the sella. Prompt transport to the angiography suite is necessary as this procedure can be both diagnostic and therapeutic. Use of external ventriculostomy and subarachnoid hemorrhage protocols can then be utilized as necessary after the patient is stabilized. In our study the diaphragm was opened to allow evacuation of the hemorrhage. The hemorrhage was controlled with irrigation and sellar packing. Stat head CT followed by diagnostic angiography and coil embolization ultimately secured the aneurysm.

In the pre-CT and MRI-era angiography was a standard part of the pre-op workup for transphenoidal surgery [18]. It was used for several reasons, such as evaluating supraellar extension, as well as the identification of vascular anomalies, therefore raising the question of pre operative vascular screening. Jakumbowski et al. recommended that while encasement of vessels by tumors is rare, the number of vascular abnormalities warrants pre-op angiography [2]. Non invasive procedures such as CTA and MRA have been shown to have equivalent sensitivities to angiography for aneurysms measuring at least $3 \mathrm{~mm}$ [19] and may represent an alternative to formal angiography in preoperative planning. At this time we cannot recommend preoperative screening for all pituitary adenomas in the absence of other risk factors for vascular abnormalities due to the rarity of remote rupture of aneurysms and the current cost of imaging studies. Adding to our recommendation against screening preoperatively is the ensuing management of the newly identified small un-ruptured remote aneurysms. The confounding nature of small un-ruptured aneurysms is not a new problem and has been described in other surgical procedures such as Carotid Endarterectomy [20]. Small, unruptured, intracranial aneurysms probably should not be a contraindication to transphenoidal surgery, but the patient should be informed there is a possibility of rupture during the procedure, which could result in higher morbidity and mortality.

\section{Conclusion}

We have reported the first case of remote intra-operative posterior circulation aneurismal rupture during routine endoscopic transphenoidal surgery for a pituitary macroadenoma. Preoperative diagnosis of IA could provide benefit, but evidence is not strong enough to support routine preoperative angiographic screening without identifying any other significant risk factor for IA. Intra-operative identification of aneurismal rupture is important, as continued bleeding and re-rupture can lead to poor outcomes. Identification in this case was possible by viewing a tense, red diaphragm sella. Intra-operative management consists of prompt recognition, irrigation, sellar packing and blood transfusion if necessary. Diagnosis is made with emergent angiography and early treatment with endovascular or open surgical technique is necessary to minimize morbidity.

\section{Conflicts of Interest}

The authors declare no conflicts of interest. This case was not previously published or presented at National Meetings.

\section{References}

[1] Wakai, S., Fukushima, T., Furihata, T. and Sano, K. (1979) Association of Cerebral Aneurysm with Pituitary Adenoma. Surgical Neurology, 12, 503-507.

[2] Jakubowski, J. and Kendall, B. (1978) Coincidental Aneurysms with Tumours of Pituitary Origin. Journal of Neurology. Neurosurgery \& Psychiatry, 41, 972-979. http://dx.doi.org/10.1136/jnnp.41.11.972

[3] Pant, B., Arita, K., Kurisu, K., Tominaga, A., Eguchi, K. and Uozumi, T. (1997) Incidence of Intracranial Aneurysm Associated with Pituitary Adenoma. Neurosurgical Review, 20, 13-17. http://dx.doi.org/10.1007/BF01390519

[4] Wang, C.-S., Yeh, T.-C., Wu, T.-C. and Yeh, C.-H. (2009) Pituitary Macroadenoma Co-Existent with Supraclinoid Internal Carotid artery Cerebral Aneurysm: A Case Report and Review of the Literature. Cases Journal, 2, 6459. http://dx.doi.org/10.4076/1757-1626-2-6459

[5] Ezzat, S., Asa, S.L., Couldwell, W.T., Barr, C.E., Dodge, W.E., Vance, M.L. and McCutcheon, I.E. (2004) The Prevalence of Pituitary Adenomas: A Systematic Review. Cancer, 101, 613-619. http://dx.doi.org/10.1002/cncr.20412

[6] Locatelli, M., Spagnoli, D., Caroli, M., Isalberti, M., Branca, V., Gaini, S.M. and Lania, A. (2008) A Potential Catas- 
trophic Trap: An Unusually Presenting Sellar Lesion. European Journal of Neurology, 15, 98-101.

[7] Oh, M.C., Kim, E.H. and Kim, S.H. (2012) Coexistence of Intracranial Aneurysm in 800 Patients with Surgically Confirmed Pituitary Adenoma. Journal of Neurosurgery, 116, 942-947. http://dx.doi.org/10.3171/2011.12.JNS11875

[8] du Boulay, G.H. (1965) Some Observations on the Natural History of Intracranial Aneurysms. British Journal of Radiology, 38, 721-757. http://dx.doi.org/10.1259/0007-1285-38-454-721

[9] van Lindert, E.J., Böcher-Schwarz, H.G. and Perneczky, A. (2001) The Influence of Surgical Experience on the Rate of Intraoperative Aneurysm Rupture and Its Impact on Aneurysm Treatment Outcome. Surgical Neurology, 56, 151-156. http://dx.doi.org/10.1016/S0090-3019(01)00547-X

[10] Stoodley, M.A., Macdonald, R.L. and Weir, B.K. (1998) Pregnancy and Intracranial Aneurysms. Neurosurgery Clinics of North America, 9, 549-556.

[11] Horsley, M., Taylor, T.K. and Sorby, W.A. (1997) Traction-Induced Rupture of an Extracranial Vertebral Artery Aneurysm Associated With Neurofibromatosis: A Case Report. Spine, 22, 225-227. http://dx.doi.org/10.1097/00007632-199701150-00019

[12] Sahjpaul, R.L., Abdulhak, M.M., Drake, C.G. and Hammond, R.R. (1998) Fatal Traumatic Vertebral Artery Aneurysm Rupture. Journal of Neurosurgery, 89, 822-824. http://dx.doi.org/10.3171/jns.1998.89.5.0822

[13] Tsuchida, T., Tanaka, R., Yokoyama, M. and Sato, H. (1983) Rupture of Anterior Communicating Artery Aneurysm during Transsphenoidal Surgery for Pituitary Adenoma. Surgical Neurology, 20, 67-70. http://dx.doi.org/10.1016/0090-3019(83)90110-6

[14] Suzuki, H., Muramatsu, M., Murao, K., Kawaguchi, K. and Shimizu, T. (2001) Pituitary Apoplexy Caused by Ruptured Internal Carotid Artery Aneurysm. Stroke, 32, 567-569. http://dx.doi.org/10.1161/01.STR.32.2.567

[15] Berker, M. (2010) Overview of Vascular Complications of Pituitary Surgery with Special Emphasis on Unexpected Abnormality. Pituitary, 13, 160-167. http://dx.doi.org/10.1007/s11102-009-0198-7

[16] Paré, L., Delfino, R. and Leblanc, R. (1992) The Relationship of Ventricular Drainage to Aneurysmal Rebleeding. Journal of Neurosurgery, 76, 422-427.

[17] Houkin, K. (1999) Intra-Operative Premature Rupture of the Cerebral Aneurysms. Analysis of the Causes and Management. Acta Neurochirurgica, 141, 1255-1263. http://dx.doi.org/10.1007/s007010050428

[18] Liu, J.K., Das, K., Weiss, M.H., Laws, E.R. and Couldwell, W.T. (2001) The History and Evolution of Transsphenoidal Surgery. Journal of Neurosurgery, 95, 1083-1096. http://dx.doi.org/10.3171/jns.2001.95.6.1083

[19] Karamessini, M.T., Kagadis, G.C., Petsas, T., Karnabatidis, D., Konstantinou, D., Sakellaropoulos, G.C., Nikiforidis, G.C. and Siablis, D. (2004) CT Angiography with Three-Dimensional Techniques for the Early Diagnosis of Intracranial Aneurysms. Comparison with Intra-Arterial DSA and the Surgical Findings. European Journal of Radiology, 49, 212-223. http://dx.doi.org/10.1016/S0720-048X(03)00173-6

[20] Kappelle, L.J. (2000) Small, Unruptured Intracranial Aneurysms and Management of Symptomatic Carotid Artery Stenosis. Neurology, 55, 307-309. http://dx.doi.org/10.1212/WNL.55.2.307

\section{Abbreviations}

IA (Intracranial Aneurysm) 